Association for

Computing Machinery

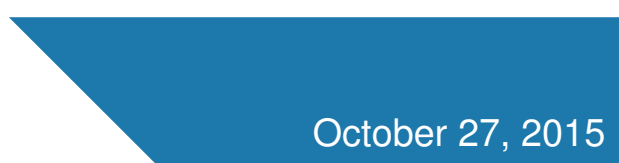

Pittsburgh, PA, USA

Advancing Computing as a Science \& Profession

\title{
SEPS 15
}

Proceedings of the 2nd International Workshop on

\section{Software Engineering for Parallel Systems}

Edited by:

Ali Jannesari, Siegfried Benkner, Xinghui Zhao, Ehsan Atoofian, and Yukionri Sato

Sponsored by:

ACM SIGPLAN

Supported by:

Microsoft, Oracle, NSF, IBM Research, Samsung, HP, Google, Facebook, Intel, Huawei, DePaul University, Royal Society Publishing 


\section{The Association for Computing Machinery, Inc. 2 Penn Plaza, Suite 701 New York, NY 10121-0701}

Copyright (C) 2015 by the Association for Computing Machinery, Inc (ACM). Permission to make digital or hard copies of portions of this work for personal or classroom use is granted without fee provided that the copies are not made or distributed for profit or commercial advantage and that copies bear this notice and the full citation on the first page. Copyrights for components of this work owned by others than ACM must be honored. Abstracting with credit is permitted.

To copy otherwise, to republish, to post on servers or to redistribute to lists, requires prior specific permission and/or a fee. Request permission to republish from: Publications Dept. ACM, Inc.

Fax +1-212-869-0481 or E-mail permissions@acm.org.

For other copying of articles that carry a code at the bottom of the first or last page, copying is permitted provided that the per-copy fee indicated in the code is paid through the Copyright Clearance Center, 222 Rosewood Drive, Danvers, MA 01923.

\section{Notice to Past Authors of ACM-Published Articles}

ACM intends to create a complete electronic archive of all articles and/or other material previously published by ACM. If you have written a work that was previously published by ACM in any journal or conference proceedings prior to 1978, or any SIG Newsletter at any time, and you do NOT want this work to appear in the ACM Digital Library, please inform permissions@acm.org, stating the title of the work, the author(s), and where and when published.

ACM ISBN: 978-1-4503-3910-0

Additional copies may be ordered prepaid from:

$\begin{array}{ll}\text { ACM Order Department } & \text { (U.S.A. and Canada) } \\ \text { P.O. BOX 11405 } & +1-212-626-0500 \\ \text { Church Street Station } & \text { (All other countries) } \\ \text { New York, NY 10286-1405 } & \text { Fax: +1-212-944-1318 } \\ & \text { E-mail: acmhelp@acm.org }\end{array}$

Production: Conference Publishing Consulting

D-94034 Passau, Germany, info@conference-publishing.com 


\section{Message from the Chairs}

Welcome to the second international workshop on Software Engineering for Parallel Systems (SEPS) held in Pittsburgh, PA, USA on October 27, 2015 and co-located with the ACM SIGPLAN conference on Systems, Programming, Languages and Applications: Software for Humanity (SPLASH 2015). The purpose of this workshop is to provide a stable forum for researchers and practitioners dealing with compelling challenges of the software development life cycle on modern parallel platforms.

The increased complexity of parallel applications on modern parallel platforms (e.g. multicore, manycore, distributed or hybrid) requires more insight into development processes, and necessitates the use of advanced methods and techniques supporting developers in creating parallel applications or parallelizing and reengineering sequential legacy applications. We aim to advance the state of the art in different phases of parallel software development, covering software engineering aspects such as requirements engineering and software specification; design and implementation; program analysis, profiling and tuning; testing and debugging.

The format of the workshop will be a full-day mini-conference. We welcome original, unpublished regular papers (10 pages) on current research, and industrial papers and tool presentations (short papers, 4 pages - excluding references). We publish accepted papers as post-proceedings in the ACM Digital Library. For organizing the technical sessions, the Program Committee reviewed all the submitted papers professionally. We would like to express our sincere thanks to all the members of the Program Committee.

All submitted papers received in average 4 reiews (at least three reviews), and we selected 9 excellent papers. Authors of each accepted paper will have $30 \mathrm{~min}$ (including $5 \mathrm{~min}$ discussion) to present their work. The workshop is also enriched by a invited talk, titled "Hybrid Inference of Semantics for Software Adaptation" by Dr. Karl Palmskog (University of Illinois at Urbana-Champaign).

I also happily thank all the Organizing Committee members who helped me make this workshop a success. Ehsan Atoofian (Lakehead University), Siegfried Benkner (University of Vienna), Yukinori Sato (Tokyo Institute of Technology), Xinghui Zhao (Washington State University). I would like to express my sincere appreciation for their significant efforts to make the workshop happen.

Finally, we would like to thank the speakers of this workshop and all attendees. We hope you will enjoy the SEPS 2015.

\section{Ali Jannesari}

SEPS 2015, Primary Organizer, Organizing Committee

Technical University of Darmstadt 


\section{SEPS 2015 Organization}

Organizing Committee

\section{Primary Organizer}

Ali Jannesari

\section{Members}

Ehsan Atoofian

Siegfried Benkner

Yukinori Sato

Xinghui Zhao

Program Committee

Jeremy Bradbury

Jeffrey Carver

Toshio Endo

Clemens Grelck

Takahiro Katagiri

Christoph Kessler

Victor Lee

Zhiyuan Li

Hiroko Midorikawa

Pablo Oliveira

Miquel Pericàs

Michael Philippsen

Michael Pradel

Ricardo Rocha

Bernhard Rumpe

Hiroyuki Takizawa

Masaaki Terai

Massimo Torquati

Shmuel Ur

Josef Weidendorfer
Technical University of Darmstadt, Germany

Lakehead University, Canada

University of Vienna, Austria

Tokyo Institute of Technology, Japan

Washington State University, USA

University of Ontario Institute of Technology, Canada

University of Alabama, USA

Tokyo Institute of Technology, Japan

University of Amsterdam, Netherlands

University of Tokyo, Japan

Linköping University, Sweden

Intel, CA, USA

Purdue University, USA

Seikei University, Japan

University of Versailles, France

Tokyo Institute of Technology, Japan

University of Erlangen-Nuremberg, Germany

Technical University of Darmstadt, Grrmany

University of Porto, Portugal

RWTH Aachen University, Germany

Tohoku University, Japan

RIKEN, Japan

University of Pisa, Italy

Ur Innovation, Israel

Technical University of Munich, Germany 


\section{Contents}

\section{Frontmatter}

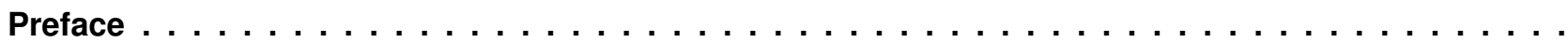

\section{Profiling and Program Analysis}

Exana: An Execution-Driven Application Analysis Tool for Assisting Productive Performance Tuning Yukinori Sato, Shimpei Sato, and Toshio Endo - Tokyo Institute of Technology, Japan . . . . . . . . . . . . . . .

Profiling for Detecting Performance Anomalies in Concurrent Software

Faheem Ullah and Thomas R. Gross - ETH Zurich, Switzerland . . . . . . . . . . . . . . . . . . . . . . .

Annotatable Systrace: An Extended Linux ftrace for Tracing a Parallelized Program

Daichi Fukui, Mamoru Shimaoka, Hiroki Mikami, Dominic Hillenbrand, Hideo Yamamoto, Keiji Kimura, and Hironori

Kasahara — Waseda University, Japan . . . . . . . . . . . . . . . . . . . . . . . . .

\section{Modeling Techniques for Parallel Software}

Interleaving Generation for Data Race and Deadlock Reproduction

Luis M. Carril and Walter F. Tichy — KIT, Germany . . . . . . . . . . . . . . . . . . .

An Empirical Study on Parallelism in Modern Open-Source Projects

Marc Kiefer, Daniel Warzel, and Walter F. Tichy - KIT, Germany . . . . . . . . . . . . . . . . . . . 35

ATL-MR: Model Transformation on MapReduce

Amine Benelallam, Abel Gómez, and Massimo Tisi — AtlanMod, France; University of Zaragoza, Spain . . . . . .

\section{Performance Tuning and Auto-tuning}

Investigating Potential Performance Benefits of Memory Layout Optimization Based on Roofline Model Shimpei Sato, Yukinori Sato, and Toshio Endo - Tokyo Institute of Technology, Japan . . . . . . . . . . . . . . . .

Empirical Performance Study of Speculative Parallel Processing on Commercial Multi-core CPU with Hardware Transactional Memory

Kanemitsu Ootsu, Yutaka Matsuno, Takeshi Ohkawa, Takashi Yokota, and Takanobu Baba - Utsunomiya University, Japan; Mitsubishi Electric Information Network, Japan . . . . . . . . . . . . . . . . . . . . . . . . . .

Lighthouse: A Taxonomy-Based Solver Selection Tool

Kanika Sood, Boyana Norris, and Elizabeth Jessup - University of Oregon, USA; University of Colorado at Boulder,

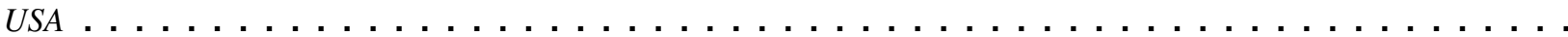

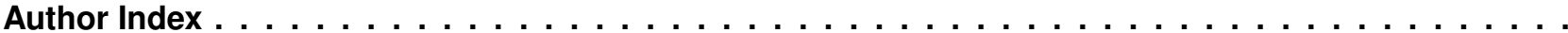

